\title{
Utilidad del índice UDT-65 en adultos con dolor torácico y electrocardiograma normal o no diagnóstico
}

\section{Usefulness of the CPU-65 index in adults with chest pain and normal or non-diagnostic electrocardiogram}

\author{
Ella C. Roncallo ${ }^{1 *}$, Álvaro J. Ruiz², Erick R. Solano ${ }^{3}$ y Nelcy Rodríguez-Malagón ${ }^{1}$
}

${ }^{1}$ Departamento de Epidemiología Clínica y Bioestadística, Facultad de Medicina, Pontificia Universidad Javeriana; ${ }^{2}$ Departamento de Medicina Interna, Facultad de Medicina, Pontificia Universidad Javeriana; ${ }^{3}$ Departamento de Urgencias, Fundación Clínica Shaio. Bogotá, Colombia

\section{Resumen}

Objetivo: Evaluar la utilidad del índice UDT-65 para la estratificación del dolor torácico en urgencias en una población colombiana en la que se sospecha enfermedad coronaria. Método: Se condujo la validación externa del índice UDT-65 en una cohorte concurrente que incluyó pacientes que ingresaron a urgencias de una clínica cardiovascular en Bogotá con dolor torácico no traumático, y electrocardiograma normal o no diagnóstico. Se evaluaron 1320 pacientes de 18 o más años y se determinó la utilidad del índice en términos de calibración (uso de gráfico, ji al cuadrado para datos agrupados y prueba de bondad de ajuste de Hosmer-Lemeshow) y de capacidad de discriminación del modelo (curva de características operativas del receptor [ROC] y área bajo ella [AUC]). Resultados: El índice UDT-65 en esta población suministró evidencia de su utilidad en términos de calibración y capacidad de discriminación, para efectuar una buena aplicación de él en aquellos pacientes que consulten al servicio de urgencias de una clínica cardiovascular por dolor torácico no traumático de posible origen coronario. La capacidad de discriminación del índice UDT-65 fue adecuada, pues con un área bajo la curva ROC de 0.867 (IC 95\% 0-847-0.885), que se acerca al valor obtenido (AUC 0.87) en la población española en que se desarrolló el índice. Conclusiones: Se necesitan más estudios similares en otras instituciones, dado el buen resultado, en beneficio de más pacientes.

Palabras clave: Calibración. Discriminación. Enfermedad coronaria.

\section{Abstract}

Objective: To evaluate the clinical usefulness, in the emergency service, of the UDT-65 index for chest pain stratification in colombian population with suspected coronary disease. Method: The external validation of the UDT-65 index was conducted in a concurrent cohort that included patients admitted to the emergency service of the cardiovascular clinic in Bogotá with non-traumatic chest pain and normal or non-diagnostic electrocardiogram. 1320 patients were evaluated and the usefulness of this instrument was determined in terms of calibration (use of graph, Chi-square test for group data and the Hosmer-Lemeshow goodness-of-fit test) and discrimination capacity of the model (curve of receiver operating characteristics [ROC] and by finding the area under the curve [AUC]). Results: The UDT-65 index in the population under study, provided evidence of its usefulness in terms of calibration and discrimination capacity; this, in pursuance of a good application of the instrument in those patients who consult the Emergency Department of the cardiovascular clinic for non-traumatic chest pain of possible

\section{Correspondencia:}

*Ella C. Roncallo

E-mail: eroncallo@javeriana.edu.co; ellaroncallo@gmail.com
Disponible en internet: 29-11-2021 Rev Colomb Cardiol. 2021;28(5):410-420 www.rccardiologia.com 0120-5633 / C 2020 Sociedad Colombiana de Cardiología y Cirugía Cardiovascular. Publicado por Permanyer. Este es un artículo open access bajo la licencia CC BY-NC-ND (http://creativecommons.org/licenses/by-nc-nd/4.0/). 
coronary origin. The discrimination capacity of the UDT65 index was adequate, with an area under the ROC curve of 0.867 (95\% Cl 0.847-0.885), which is close in value to the one obtained (AUC 0.87) in the Spanish population where the original index was developed. Conclusions: More similar studies are needed in other institutions, due to the excellent and beneficial outcomes.

Key words: Calibration. Discrimination. Coronary disease.

\section{Introducción}

La enfermedad coronaria es la causa principal de mortalidad en la mayoría de los países industrializados ${ }^{1}$. El dolor torácico es la manifestación clínica más frecuente de la enfermedad coronaria y representa uno de los mayores motivos de consulta a los servicios de urgencias.

El enfoque clínico de los pacientes que acuden a urgencias con dolor torácico que supone un origen coronario representa un reto diagnóstico, debido a que, si bien representa entre el $5 \%$ y el $20 \%$ de todas las urgencias, el diagnóstico solo se confirma en menos del $50 \%$ de los $\operatorname{casos}^{2,3}$, hecho que ocasiona un incremento en el número de ingresos hospitalarios y en los costos de la atención, que generan en los Estados Unidos de América un costo anual cercano a los 12 billones de dólares ${ }^{4,5}$. Por otro lado, entre el $2 \%$ y el $10 \%$ de los pacientes que son dados de alta desde urgencias, por considerar que el dolor no es de origen coronario, presentan un infarto agudo de miocardio, lo cual aumenta la mortalidad extrahospitalaria ${ }^{3,4}$.

En Colombia, la enfermedad coronaria es la principal causa de muerte ${ }^{6}$, y aunque no se encuentran estudios disponibles en cuanto a la frecuencia del dolor torácico en los servicios de urgencias, según datos estadísticos suministrados por la gerencia de urgencias de la Fundación Clínica Shaio, entre enero y julio de 2011 se atendieron en el servicio de urgencias de esta institución 40,030 pacientes, de los cuales el 12\% eran pacientes con dolor torácico, y de estos, solo el $25 \%$ fueron hospitalizados.

Teniendo en cuenta la magnitud del problema asistencial, el paciente con dolor torácico genera incertidumbre tanto en el médico como en él mismo y su familia. En los últimos años, la estratificación del riesgo ha adquirido relevancia al ser considerada como la base para la toma de decisiones médicas. Con la incorporación de las técnicas estadísticas a la investigación clínica se han desarrollado múltiples modelos para predecir el pronóstico de los pacientes coronarios, y dichos modelos han evolucionado hasta ser más exactos, y algunos poco prácticos ${ }^{7}$, pero aún no logran resolver el problema. Por esta razón, en un intento por mejorar las técnicas de predicción y diagnóstico, todavía se desarrollan modelos pronósticos y diagnósticos.

Diseñar un modelo de predicción útil no es tarea fácil ${ }^{8,9}$. Esta estrategia requiere un número elevado de pacientes que representen la condición clínica de interés. Así mismo, el número de desenlaces debe ser suficiente para permitir un cálculo estadístico adecuado, las variables clínicas deben ser relevantes, los desenlaces deben ser clínicamente importantes y el modelo tiene que ser probado en una población diferente de la población de estudio en la que fue desarrollado ${ }^{10}$.

El índice UDT-65 ha sido validado en población española y su capacidad de discriminación para clasificar el diagnóstico final de enfermedad coronaria fue adecuada $^{11}$. Es conveniente evaluar en nuestro medio la utilidad del índice UDT-65 para clasificar a los pacientes con síndrome coronario agudo asociado con enfermedad coronaria, por considerar que relaciona un menor número de variables en comparación con otras escalas, evalúa las características del dolor y requiere poco tiempo, y aunque ha sido referenciado en otros estudios europeos, no cuenta aún con validación formal en contextos diferentes del español, incluyendo el colombiano.

\section{Descripción del índice UDT-65}

En 2005, Martínez-Sellés, et al. ${ }^{12}$ llevaron a cabo una investigación cuyo objetivo era estudiar qué variables clínicas podrían estar relacionadas de manera independiente con enfermedad coronaria, en pacientes ingresados en la unidad de dolor torácico (UDT) de un hospital terciario, y secundariamente para detectar eventos coronarios en la UDT y en su seguimiento. En la metodología empleada en su estudio, evaluaron 379 pacientes que ingresaron de forma consecutiva en la UDT del Hospital Universitario Gregorio Marañón, de Madrid (España) entre julio de 2003 y septiembre de 2004, con electrocardiograma normal o no diagnóstico.

Se desarrolló un modelo logístico multivariado con inclusión de las siguientes variables clínicas: edad mayor de 64 años, sexo, factores de riesgo cardiovascular, 
antecedentes cardiovasculares, dolor torácico típico, uso de ácido acetilsalicílico, cifras de presión arterial al ingreso y frecuencia cardiaca. En el análisis multivariado se confirmó que la presencia de dolor típico anginoso, diabetes, uso de ácido acetilsalicílico y edad mayor de 64 años aumentaba de dos a tres veces la probabilidad de tener enfermedad coronaria en pacientes con dolor torácico sospechoso de síndrome coronario agudo y electrocardiograma normal o no diagnóstico ${ }^{12}$.

Con estas variables independientes se construyó el índice, y al combinarlas se encontró una relación directa con la aparición de eventos coronarios durante la estancia en la UDT, que osciló entre el $2.2 \%$ y el 14.3\% según el puntaje obtenido. Asimismo, el índice se relacionó con la presencia de enfermedad coronaria, que varió entre el $15 \%$ y el $66.7 \%$ en los puntajes de 1 a 4 del UDT-65 ${ }^{11}$.

Así las cosas, el índice UDT-65 es un instrumento construido a partir de un modelo logístico, que permite detectar o clasificar a los pacientes con baja probabilidad de enfermedad coronaria que consultan al servicio de urgencias por dolor torácico y sospecha de síndrome coronario agudo en presencia de electrocardiograma normal o no diagnóstico.

El índice UDT-65 (Tabla 1) tiene una puntuación máxima de 4 y su punto de corte es 1 ; a su vez, utiliza la escala de dolor torácico de Geleijnse ${ }^{13}$ (Tabla 2) para medir las características del dolor torácico y discriminarlo en típico si se obtienen 6 o más puntos 0 atípico si el puntaje es menor de 6 .

\section{Método}

Se llevó a cabo un estudio de evaluación de la utilidad del índice UDT-65 mediante su validación externa en pacientes de 18 o más años que ingresaron al servicio de urgencias de la Fundación Clínica Shaio entre el 1 de agosto y el 31 de octubre del 2016 con dolor torácico no traumático y electrocardiograma normal o no diagnóstico, cuya duración del dolor fuese menor de 60 minutos y que estuviesen de acuerdo con su participación en el estudio y firmaran el consentimiento informado, debido a que los pacientes dados de alta desde urgencias requerían un seguimiento telefónico a los 30 días del egreso. Asimismo, se excluyeron aquellos pacientes con falla cardiaca o falla renal de cualquier origen, así como también aquellos con antecedentes de neoplasias, trasplante de órganos o síndromes de hipercoagulabilidad.

El proceso de recolección de la muestra consideró la inclusión consecutiva de pacientes que cumplieran
Tabla 1. Escala del dolor de Geleinjse ${ }^{13}$

\begin{tabular}{|c|c|}
\hline $\begin{array}{l}\text { Localización } \\
\text { Retroesternal } \\
\text { Precordial } \\
\text { Cuello, mandíbula o epigastrio } \\
\text { Apical (debajo de la mamila izquierda) }\end{array}$ & $\begin{array}{l}+3 \\
+2 \\
+1 \\
-1\end{array}$ \\
\hline $\begin{array}{l}\text { Irradiación } \\
\text { Uno de los dos brazos } \\
\text { Hombro, espalda, cuello, mandíbula }\end{array}$ & $\begin{array}{l}+2 \\
+1\end{array}$ \\
\hline $\begin{array}{l}\text { Carácter } \\
\text { Fuertemente opresivo } \\
\text { Molestia opresiva } \\
\text { Pinchazos }\end{array}$ & $\begin{array}{l}+3 \\
+2 \\
-1\end{array}$ \\
\hline $\begin{array}{l}\text { Gravedad } \\
\text { Grave } \\
\text { Moderada } \\
\text { Varía con nitroglicerina } \\
\text { Postura } \\
\text { Respiración }\end{array}$ & $\begin{array}{l}+2 \\
+1 \\
+1 \\
-1 \\
-1\end{array}$ \\
\hline $\begin{array}{l}\text { Síntomas asociados } \\
\text { Disnea } \\
\text { Náuseas o vómitos } \\
\text { Sudación } \\
\text { Antecedentes de angina de esfuerzo }\end{array}$ & $\begin{array}{l}+2 \\
+2 \\
+2 \\
+3\end{array}$ \\
\hline
\end{tabular}

Tabla 2. Índice UDT-65

\begin{tabular}{|l|l|c|}
\hline Variable & Definición & Puntaje \\
\hline Tipicidad del dolor* & Típico ( $\geq 6$ puntos) & 1 \\
\hline Díabetes & Atico (<6 puntos) & 0 \\
\hline Sí & 1 \\
\hline Uso de ácido acetilsalicílico & So & 0 \\
\hline Edad & No & 1 \\
\hline & $\geq 65$ años & 0 \\
\hline & $<65$ años & 1 \\
\hline
\end{tabular}

*Usar escala de Geleijnse.

con los criterios de elegibilidad, cuidando de tener al menos 250 pacientes con eventos y otros 250 sin eventos ${ }^{10,14-16}$ distribuidos en todo el índice UDT-65.

Como variable de desenlace final se evaluó la presencia de enfermedad coronaria, entendida como la confirmación mediante angiografía o una prueba de detección de isquemia positiva ${ }^{17}$. Además de esta variable, se midió como variable de desenlace intermedio el síndrome coronario agudo, basado en sus definiciones operativas ${ }^{4,5,18}$. 
Durante el proceso de recolección de los datos, un primer evaluador (médico de urgencias) registró en una ficha estandarizada los datos de identificación y las características del dolor según la escala de Geleijsen. Luego, con esta información, un segundo evaluador extrajo datos de variables demográficas, como edad, sexo, raza, estado civil, procedencia, ocupación, situación laboral, nivel educativo y tipo de aseguramiento.

Se midieron las variables clínicas de interés que considera el UDT-65 (Uso de ácido acetilsalicílico, Diabetes, Tipicidad del dolor y edad de 65 años o más), y de manera adicional se midieron las variables hipertensión arterial, antecedentes familiares, colesterol total, colesterol unido a lipoproteínas de alta densidad y tabaquismo. Estas variables fueron seleccionadas para mejorar el desempeño del índice en la población de estudio, en caso de ser necesario. Su selección se basó en los resultados de los estudios INTERHEART ${ }^{19}$ y CARMELA ${ }^{20}$ realizados en Latinoamérica, en los que incluyeron a Bogotá.

Se diligenciaron 1628 formatos, de los cuales solo 1320 se consideraron aptos para el análisis. Para medir los desenlaces se definieron dos grupos: el primero conformado por los pacientes hospitalizados en quienes se determinó la presencia o no de enfermedad coronaria y el tipo de síndrome coronario agudo; el segundo, por los pacientes que fueron dados de alta desde urgencias, a los que se efectuó seguimiento telefónico a los 30 días de dicho egreso para evaluar desenlaces. Fue un cardiólogo quien midió el desenlace final.

Con el propósito de minimizar el posible sesgo de selección por clasificación errónea del dolor torácico, los médicos del servicio de urgencias fueron capacitados por un experto temático (especialista en medicina interna y cardiología) sobre las características relevantes en la interrogación del dolor torácico de origen no traumático, con énfasis en la etiología coronaria. Para el manejo del sesgo asociado a la búsqueda activa y de verificación de desenlaces, tanto el desenlace final como el intermedio fueron medidos por un cardiólogo.

\section{Análisis estadístico}

Para el procesamiento de los datos se utilizó el programa STATA 14. Inicialmente se realizó un análisis exploratorio de los datos en búsqueda de valores faltantes $^{21}$, con búsqueda activa de los datos en la historia clínica o llamando al paciente cuando fue necesario.
En el análisis descriptivo se calcularon los promedios y las desviaciones estándar para las variables cuantitativas, las frecuencias para las variables nominales, y la mediana y la moda para las variables ordinales, como el puntaje total de la escala.

Con los datos obtenidos se calcularon las estimaciones de riesgo de tener enfermedad coronaria para cada puntaje UDT-65 a partir de los coeficientes de regresión obtenidos del modelo inicial (población española). A continuación, se procedió a realizar la validación del modelo ${ }^{22,23}$ en nuestra población, en términos de desempeño (calibración y capacidad de discriminación ${ }^{24-26}$ ) y utilidad clínica (aplicabilidad del instrumento).

La calibración del modelo se evaluó mediante un gráfico de calibración, comparando los eventos esperados con los eventos observados al interior de cada grupo de puntaje del índice UDT-65. Se consideró que un modelo bien calibrado debía tener en el gráfico una pendiente (B1) de 1 y un intercepto (B0) igual a $0^{22-24}$. De igual manera, se realizó una prueba de ji al cuadrado para comparar los eventos esperados y observados, y se evaluó la bondad del ajuste con la prueba de Hosmer-Lemeshow ${ }^{25}$. Sin embargo, sin desconocer la importancia de los estadísticos, fue una decisión clínica la que definió si el modelo estaba bien calibrado, mediante el grado de acuerdo de las diferencias absolutas entre lo esperado y lo observado de un $5 \% 0$ menos $^{9,26}$.

Se evaluó la capacidad de discriminación del modelo mediante una curva ROC (Receiver Operating Characteristic) ${ }^{27,28}$ y se determinó el área bajo la curva (AUC), considerando en nuestra población que el índice tendría una adecuada discriminación si el AUC era $\geq 0.80$.

El estudio se ajustó a los principios éticos consagrados en la Declaración de Helsinki ${ }^{29}$ y a la resolución No. 8430 de $1993^{30}$. Respecto a la utilización del índice UDT-65 y la escala de dolor torácico de Geleijnse, se solicitó permiso para su uso a los autores, con respuesta favorable de su parte.

\section{Resultados}

\section{Población de estudio}

De agosto a octubre de 2016 ingresaron 14,944 pacientes al servicio de urgencias de la clínica en mención, de los cuales 1898 consultaron por dolor torácico no traumático, lo que correspondió al $12.7 \%$ del total de las urgencias. La media diaria de pacientes con 
Tabla 3. Características clínicas de la población de estudio $(n=1320)$

\begin{tabular}{|c|c|c|c|}
\hline \multicolumn{2}{|l|}{ Características clínicas } & n & $\%$ \\
\hline \multicolumn{2}{|l|}{ Dolor torácico típico } & 735 & 55.7 \\
\hline \multicolumn{2}{|l|}{ Diabetes } & 216 & 16.4 \\
\hline \multicolumn{2}{|l|}{ Uso crónico de ácido acetilsalicílico } & 543 & 41.1 \\
\hline \multicolumn{2}{|l|}{ Tabaquismo } & 477 & 36.1 \\
\hline \multicolumn{2}{|l|}{ Hipertensión arterial } & 766 & 58 \\
\hline \multicolumn{2}{|l|}{ Dislipidemia } & 502 & 38 \\
\hline \multicolumn{2}{|l|}{ Enfermedad coronaria previa } & 417 & 31.6 \\
\hline \multicolumn{2}{|c|}{$\begin{array}{l}\text { Antecedentes familiares de enfermedad } \\
\text { coronaria }\end{array}$} & 189 & 14.3 \\
\hline Características clínicas continuas & n & Promedio & DE \\
\hline Peso (kg) & 1320 & 70.9 & 13.1 \\
\hline Talla (cm) & 1320 & 165.1 & 7.9 \\
\hline Glucemia (mg/dl) & 1248 & 97.1 & 26.8 \\
\hline Colesterol total (mg/dl) & 1058 & 168.6 & 41.3 \\
\hline Colesterol HDL (mg/dl) & 1057 & 40.1 & 9.6 \\
\hline Colesterol LDL (mg/dl) & 1043 & 99.9 & 35.6 \\
\hline Triglicéridos (mg/dl) & 1055 & 144.8 & 71.8 \\
\hline
\end{tabular}

DE: desviación estándar; HDL: lipoproteínas de alta densidad; LDL: lipoproteínas de baja densidad.

dolor torácico no traumático fue de 21 . En relación con la presencia de enfermedad coronaria, la proporción de pacientes que ingresaron con dolor torácico no traumático y presentaron la condición fue del $40.3 \%$ (533/1320), y de estos, el 64.4\% (343/533) presentaron síndrome coronario agudo (Tabla 3 ).

La tabla 4 muestra las características sociodemográficas de la población objeto de estudio. Las características clínicas de los pacientes se resumen en la tabla 3. La tabla 5 muestra la distribución del índice UDT-65, en la que se refleja claramente que la mitad de los pacientes se ubicaron entre los puntajes 0 y 1 , considerados de bajo riesgo. Las características clínicas de los pacientes según el puntaje del UDT-65 obtenido se resumen en la tabla 6 .

El índice UDT-65 se relacionó con la presencia de síndrome coronario agudo en el $1.8 \%(5 / 277)$ de los pacientes con puntaje 0 , proporción que varió hasta un $73.7 \%$ (42/57) en los pacientes con puntaje 4.

Respecto al manejo de los pacientes, 757 fueron hospitalizados y en estos la proporción de enfermedad
Tabla 4. Descripción general de las caracteristícas sociodemográficas de la población de estudio $(n=1320)$

\begin{tabular}{|l|l|c|c|}
\hline Características sociodemográficas & n & $\%$ \\
\hline Edad & Promedio \pm DE & $60.2 \pm 14.4$ & \\
\hline Sexo & Femenino & 577 & 43.7 \\
\hline Raza & Masculino & 743 & 56.3 \\
\hline & Mestiza & 1164 & 88.2 \\
\hline \multirow{2}{*}{ Seguridad social } & Régimen contributivo & 1264 & 10.6 \\
\cline { 2 - 4 } & Afrodescendiente & 13 & 0.9 \\
\hline & Régimen subsidiado & 15 & 1.1 \\
\hline & Régimen especial & 41 & 3.1 \\
\hline Medicina prepagada y seguros & 499 & 37.8 \\
\hline
\end{tabular}

DE: desviación estándar.

Tabla 5. Distribución del índice por puntaje

\begin{tabular}{|l|c|c|}
\hline Puntaje UDT-65 & $\mathbf{n}$ & $\%$ \\
\hline 0 & 277 & 20.9 \\
\hline 1 & 384 & 29.1 \\
\hline 2 & 363 & 27.5 \\
\hline 3 & 239 & 18.1 \\
\hline 4 & 57 & 4.3 \\
\hline Total & 1320 & 100 \\
\hline
\end{tabular}

coronaria fue del $60.2 \%$, mientras que en los pacientes para los que se decidió manejo ambulatorio la proporción de enfermedad coronaria fue del $13.7 \%$. En total, se decidió el alta médica desde urgencias en el $43 \%$ de los ingresos. El diagnóstico más frecuente fue dolor en el pecho no especificado.

Ningún paciente con un índice UDT-65 de 0 murió durante el seguimiento. Dos pacientes con índice de 1 murieron durante el seguimiento, ambos de sexo femenino; la primera de 55 años, sin enfermedad coronaria, hospitalizada porque presentó un síndrome coronario agudo tipo infarto agudo de miocardio sin elevación del ST secundario a una taquicardia supraventricular, y la otra de 86 años, sin enfermedad coronaria, quien murió durante el seguimiento ambulatorio. 
Tabla 6. Características clínicas por puntaje UDT-65 en 1320 pacientes con dolor torácico no traumático

\begin{tabular}{|c|c|c|c|c|c|c|}
\hline Variable & $n(\%)$ & $\begin{array}{c}\text { UDT- } 65=0 \\
n(\%)\end{array}$ & $\begin{array}{c}\text { UDT-65 = } 1 \\
n(\%)\end{array}$ & $\begin{array}{c}\text { UDT-65 = } 2 \\
n(\%)\end{array}$ & $\begin{array}{c}\text { UDT-65 = } 3 \\
n(\%)\end{array}$ & $\begin{array}{c}\text { UDT-65 }=4 \\
n(\%)\end{array}$ \\
\hline Edad $\geq 65$ años & $560(42.2)$ & 0 & $82(21.3)$ & $225(62)$ & $196(82)$ & $57(100)$ \\
\hline Mujeres & $577(43.7)$ & $124(44.8)$ & $186(48.3)$ & $160(44.1)$ & $80(33.5)$ & $27(47.4)$ \\
\hline Dolor típico & $735(55.7)$ & 0 & $236(61.5)$ & $232(64)$ & $210(87.9)$ & $57(100)$ \\
\hline Uso de ácido acetilsalicílico & $543(41.1)$ & 0 & $57(14.8)$ & $213(58.7)$ & $216(90.4)$ & $57(100)$ \\
\hline $\begin{array}{l}\text { Factores de riesgo cardiovascula } \\
\text { Tabaquismo } \\
\text { Diabetes } \\
\text { Hipertensión arterial } \\
\text { Dislipidemia } \\
\text { Antecedentes familiares }\end{array}$ & $\begin{array}{l}477(36.1) \\
217(16.4) \\
766(58.0) \\
502(38.0) \\
189(14.3)\end{array}$ & $\begin{array}{c}85(30.7) \\
0 \\
65(23.5) \\
62(22.4) \\
40(14.4)\end{array}$ & $\begin{array}{c}122(31.7) \\
10(2.6) \\
163(42.4) \\
135(35.1) \\
56(14.6)\end{array}$ & $\begin{array}{c}149(41) \\
55(15.1) \\
263(72.5) \\
173(47.7) \\
61(16.8)\end{array}$ & $\begin{array}{l}100(41.8) \\
95(39.7) \\
220(92) \\
104(43.5) \\
30(12.6)\end{array}$ & $\begin{array}{c}21(36.8) \\
57(100) \\
55(96.5) \\
28(49.1) \\
0\end{array}$ \\
\hline $\begin{array}{l}\text { Antecedentes cardiovasculares } \\
\text { Enfermedad coronaria previa } \\
\text { Insuficiencia cardiaca } \\
\text { FA crónica } \\
\text { Enfermedad arterial periférica }\end{array}$ & $\begin{array}{c}417(31.6) \\
45(3.4) \\
35(2.7) \\
37(2.8)\end{array}$ & $\begin{array}{c}1(0.4) \\
2(0.7) \\
0 \\
0\end{array}$ & $\begin{array}{c}54(14.0) \\
6(1.6) \\
9(2.3) \\
6(1.6)\end{array}$ & $\begin{array}{l}152(42) \\
14(3.9) \\
15(4.1) \\
14(3.9)\end{array}$ & $\begin{array}{c}161(67.4) \\
16(6.7) \\
6(2.5) \\
13(5.4)\end{array}$ & $\begin{array}{c}49(86) \\
7(12.3) \\
0 \\
4(7.0)\end{array}$ \\
\hline
\end{tabular}

FA: fibrilación auricular.

Tabla 7. Coeficientes de regresión del índice UDT-65 en el estudio español

\begin{tabular}{|l|c|c|}
\hline Variable & Peso aditivo & $\beta \mathbf{i}$ \\
\hline Dolor típico & 1 & 0.6419 \\
\hline Uso de ácido acetilsalicílico & 1 & 1.1632 \\
\hline Diabetes & 1 & 0.5306 \\
\hline Edad $\geq 65$ años & 1 & 0.7419 \\
\hline Constante & & -1.273 \\
\hline
\end{tabular}

\section{Evaluación del desempeño del modelo UDT-65}

Los coeficientes de regresión obtenidos del modelo original (español) derivado para el índice UDT-65 fueron utilizados para las estimaciones de las probabilidades (riesgo) de tener enfermedad coronaria en nuestra población. En el estudio español, los coeficientes de regresión calculados se muestran en la tabla 7 . En la tabla 8 se encuentran las diferentes probabilidades estimadas para cada subgrupo del índice UDT-65 en la población colombiana estudiada; se halló que la probabilidad de tener enfermedad coronaria osciló entre el $5.9 \%$ si el paciente tenía un puntaje de 0 (menor de 65 años, sin dolor típico anginoso, sin diabetes ni uso de ácido acetilsalicílico) y el $93.8 \%$ si el paciente tenía un puntaje de 4.
Tabla 8. Estimación del riesgo en la población bajo estudio según el índice UDT-65

\begin{tabular}{|l|c|}
\hline Índice UDT-65 & $P\left(E C_{\mathrm{e}}\right)$ \\
\hline Puntaje 0 & 0.059 \\
\hline Puntaje 1 & 0.238 \\
\hline Puntaje 2 & 0.565 \\
\hline Puntaje 3 & 0.833 \\
\hline Puntaje 4 & 0.938 \\
\hline
\end{tabular}

El desempeño del modelo se evaluó en términos de calibración y capacidad de discriminación. La tabla 9 muestra la calibración del índice UDT-65 en la población objeto de estudio y su comportamiento en los cinco grupos de riesgo en relación con los eventos de enfermedad coronaria observados frente a los esperados.

Se evaluó la calibración mediante un gráfico que relacionó la proporción de eventos observados frente a esperados según el índice (Fig. 1). Se determinó clínicamente el grado de acuerdo entre ellos, con diferencias absolutas entre la proporción de eventos esperados y observados menores del $2 \%$ para los puntajes UDT-65 de 1 a 4 . El puntaje 0 tuvo un comportamiento diferente al resto de los grupos, debido a que se presentó una diferencia absoluta entre las proporciones esperada y observada del $4.1 \%$. 
Tabla 9. Calibración del índice UDT-65: proporción de eventos observados frente a esperados

\begin{tabular}{|l|l|l|l|l|l|}
\hline Grupo & $\mathbf{n}$ & Eventos observados & Proporción observada (\%) & Eventos esperados & Proporción esperada (\%) \\
\hline UDT-65 = 0 & 277 & 5 & 1.81 & 16 & 5.94 \\
\hline UDT-65 = 1 & 384 & 85 & 22.1 & 92 & 24.0 \\
\hline UDT-65 = 2 & 363 & 202 & 55.7 & 205 & 56.5 \\
\hline UDT-65 = 3 & 239 & 188 & 78.7 & 199 & 83.3 \\
\hline UDT-65 $=4$ & 57 & 53 & 92.9 & 53 & 93.8 \\
\hline
\end{tabular}

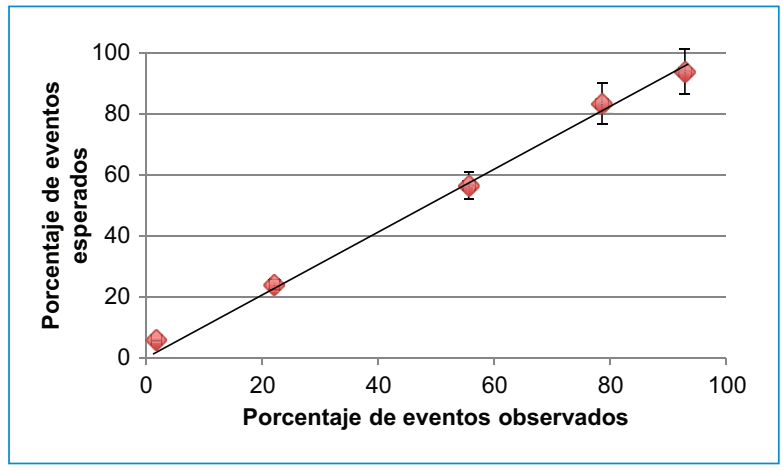

Figura 1. Calibración del modelo UDT-65: porcentaje de eventos (enfermedad coronaria) esperados frente a observados.

La prueba ji al cuadrado para comparar la proporción de eventos esperados y observados se calculó usando un nivel $\alpha$ de 0.05 y se obtuvieron los siguientes resultados: $=3.302$ con un valor $p=0.508$. La anterior es una prueba global, pero también se evaluó la bondad del ajuste con la prueba de Hosmer-Lemeshow, que mostró un resultado de 12.4 con $p=0.47$.

La capacidad de discriminación del índice UDT-65 en nuestra población de estudio mostró un AUC ROC de 0.8667 (intervalo de confianza del 95\% [IC95\%]: 0.84741-0.88543) (Fig. 2). La sensibilidad, la especificidad y los valores predictivos para diferentes puntos de corte se muestran en la tabla 10.

La razón de disparidad (OR) de enfermedad coronaria según el índice UDT-65, tanto para la población en la que fue desarrollado como para la población bajo estudio, se encuentra en la tabla 11.

\section{Utilidad clínica del índice UDT-65}

Además de la evaluación de la calibración y la discriminación, se determinó que el tiempo requerido para diligenciar el índice fue menor de 6 minutos (desviación
Tabla 10. Sensibilidad, especificidad y valores predictivos para diferentes puntos de corte del índice UDT-65

\begin{tabular}{|c|c|c|c|}
\hline & $\begin{array}{l}\text { Punto de } \\
\text { corte } 1^{*}\end{array}$ & $\begin{array}{l}\text { Punto de } \\
\text { corte } 2^{\dagger}\end{array}$ & $\begin{array}{l}\text { Punto de } \\
\text { corte } 3^{\ddagger}\end{array}$ \\
\hline Sensibilidad (\%) & 97.2 & 81 & 44.6 \\
\hline Especificidad (\%) & 34.6 & 73 & 94.1 \\
\hline $\begin{array}{l}\text { Valor predictivo positivo } \\
(\%)\end{array}$ & 52.6 & 69.3 & 85.1 \\
\hline $\begin{array}{l}\text { Valor predictivo negativo } \\
(\%)\end{array}$ & 94.2 & 83.6 & 69.4 \\
\hline $\begin{array}{l}\text { Razón de verosimilitud } \\
\text { positiva }\end{array}$ & 1.49 & 3 & 7.69 \\
\hline $\begin{array}{l}\text { Razón de verosimilitud } \\
\text { negativa }\end{array}$ & 0.08 & 0.26 & 0.59 \\
\hline
\end{tabular}

estándar: \pm 1.5$)$ y no hubo dificultad en la aplicación del instrumento.

\section{Discusión}

En este estudio realizado en una población de pacientes que ingresaron con dolor torácico no traumático a un servicio de urgencias de una institución cardiovascular de referencia nacional, el índice UDT-65 pudo ser considerado un instrumento útil como predictor del tipo diagnóstico para enfermedad coronaria o síndrome coronario agudo, toda vez que luego de efectuarse la validación externa de dicho índice mostró una buena calibración y una adecuada discriminación en la población bajo estudio. Este modelo logístico, de fácil aplicación en un servicio tan dinámico como el de urgencias, puede tener un alto valor al momento de priorizar aquellos pacientes que requieren atención 
Tabla 11. Razón de disparidad de enfermedad coronaria en el estudio español en comparación con la población colombiana estudiada

\begin{tabular}{|l|c|c|}
\hline Variable & UDT-65 español & $\begin{array}{c}\text { Población de } \\
\text { estudio }\end{array}$ \\
\hline Dolor típico & OR (IC95\%) & OR (IC95\%) \\
\hline Ácido acetilsalicílico & $1.9(1.0-3.8)$ & $2.54(1.9-3.4)$ \\
\hline Diabetes & $3.2(1.7-6.2)$ & $9.9(7.5-13.1)$ \\
\hline Edad $\geq 65$ años & $1.7(1.0-2.7)$ & $2.5(1.7-3.6)$ \\
\hline
\end{tabular}

IC95\%: intervalo de confianza del 95\%; OR: odds ratio.

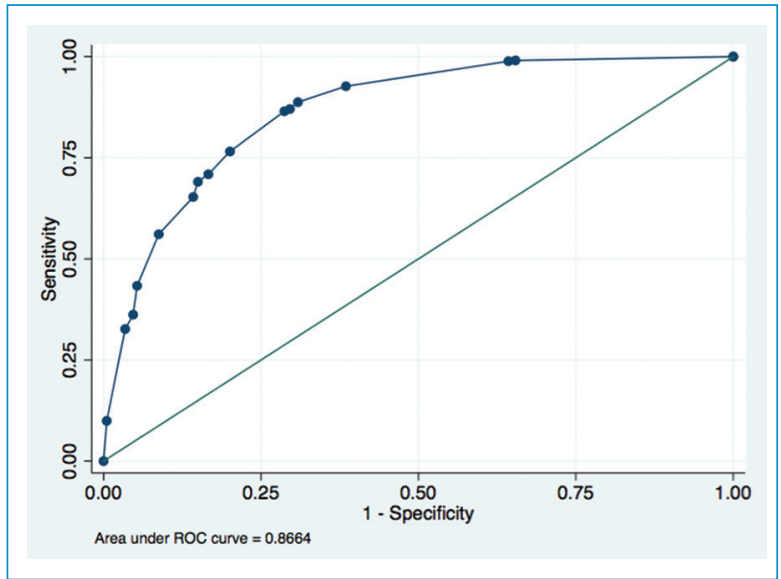

Figura 2. Capacidad discriminativa del índice UDT65. Curva ROC.

inmediata, pero que, al ser de bajo riesgo coronario, son desestimados a su ingreso.

El índice UDT-65 funcionó tanto en el grupo de pacientes ingresados como en los que fueron dados de alta al domicilio. Esta es la primera validación externa del índice UDT-65 en una población colombiana y ha permitido, a su vez, realizar la caracterización del perfil de pacientes con dolor torácico de posible origen coronario que asisten a un servicio de urgencias de una clínica especializada.

Dada la magnitud del problema asistencial, que trasciende desde lo médico-legal y ético hasta lo económico, el paciente con dolor torácico genera incertidumbre tanto en el médico como en él mismo y su familia. Por esto, la toma de decisiones médicas, sobre todo en nuestro contexto social como país en vía de desarrollo, debe ser segura y costo-efectiva, considerando el evidente envejecimiento de la población y el aumento de la carga de las enfermedades de las patologías cardiovasculares ${ }^{31}$.

La población objeto de este estudio proviene de una institución cardiovascular que es referente nacional, y en su mayoría (96\%) pertenece al régimen contributivo del Sistema de Seguridad Social, y de estos, cerca del $40 \%$ tienen medicina prepagada o un seguro médico (en razón del carácter privado de la institución), lo que implica mayor oportunidad en la atención y la posibilidad de acceder sin barreras a sus servicios, con la consiguiente baja migración a otras instituciones de salud; hecho que podría explicar la alta prevalencia de enfermedad coronaria $(40.3 \%)$ en la institución y, asimismo, la elevada frecuencia de enfermedad coronaria previa en comparación con el diagnóstico de primera vez de esta.

Es de anotar que, en la población española en la que se desarrolló el índice UDT-65, la población de 65 años o más correspondió al $48 \%$, en contraste con una menor proporción de estas edades en nuestra población $(42 \%)$. Es interesante el comportamiento de la prevalencia de factores de riesgo como el tabaquismo, que en población española fue del $49 \%$ y en la nuestra del $36 \%$, diferencia que radica en la mayor proporción de fumadores activos españoles, del $21 \%$, comparado con el $7 \%$ de fumadores activos en nuestra población; sin embargo, ambos hallazgos no logran superar la prevalencia de tabaquismo del $53 \%$ encontrada en el estudio ASPECT $^{32}$ realizado en población asiática. Llama la atención este hallazgo de tabaquismo del $36 \%$ en la población fuente, en comparación con una menor prevalencia encontrada en otros estudios colombianos como el de Bedoya, et al. ${ }^{33}$ (12.9\%) y el de Muñoz, et al. $^{23}(8.2 \%)$.

De igual forma, la prevalencia de diabetes mellitus (16.4\%) en la población bajo estudio es relativamente inferior a la encontrada en la población en la que se desarrolló el índice UDT-65 (18.6\%), y a la encontrada por Bedoya, et al. ${ }^{33}$ (21.8\%) en Armenia (Quindío), lo que contrasta con la menor prevalencia hallada en el estudio CARMELA ${ }^{20}$ en población general de Bogotá, que fue del $8.1 \%$, o en el estudio de Muñoz, et al. ${ }^{23}$, del $5.4 \%$. Es importante aclarar que las diferencias observadas en las frecuencias en nuestro estudio respecto al estudio CARMELA se deben al tipo de población incluida en cada uno: población general en el CARMELA y población que consulta a urgencias con posibilidad de enfermedad coronaria en el nuestro. Sin embargo, es relevante mencionar dicho estudio porque constituye un referente de estimaciones confiables de 
la prevalencia de factores de riesgo cardiovascular en Latinoamérica $^{20}$.

Factores de riesgo como la hipertensión arterial y la dislipidemia presentaron una alta prevalencia tanto en la población española como en la colombiana, asociado a la alta frecuencia de enfermedad coronaria previa en ambas poblaciones (42 vs. $31.6 \%$, respectivamente), resultados que en nuestro caso pueden ser explicados por el tipo de población en la que se desarrolló este estudio al tratarse de una clínica especializada en el área cardiovascular. Sin embargo, estos hallazgos contrastan con la distribución heterogénea de dichos factores de riesgo en Latinoamérica ${ }^{20,31,34-37}$, aunque esto puede deberse, según lo asumen los autores del estudio CARMELA, a una subestimación de las prevalencias en dicho estudio. A pesar de lo anterior, la población objeto de estudio tiene al menos un $40 \%$ de pacientes de bajo riesgo coronario, cuya representación permite que los resultados sean aplicables a pacientes que acuden a cualquier servicio de urgencias en nuestro medio.

Este estudio evaluó la validez externa del índice UDT-65 en población colombiana, pero no solo contempla su utilidad en función de la calibración del modelo y de su capacidad de discriminación mediante la evaluación de las características operativas del receptor, sino que rescata su utilidad en términos de tiempo, necesidad de entrenamiento y facilidad de calificación del instrumento ${ }^{38}$, hechos que hacen factible la aplicación del índice en las condiciones reales de un servicio tan dinámico como el de urgencias.

En los últimos años, con la incorporación de métodos estadísticos a la investigación clínica, se han desarrollado múltiples modelos para predecir el pronóstico de los pacientes coronarios ${ }^{7,39-43}$; desafortunadamente, muy pocos modelos predictores son de tipo diagnóstico y, sin diferenciar el tipo de modelo, son escasos los que se validan en una población diferente de la que les dio origen. En 2016 se publicó un metaanálisis en el que se analizaron 363 modelos de predicción cardiovascular y se encontró que el $64 \%$ no tenían validez externa ${ }^{44}$.

Al evaluar la calibración del modelo $0^{45,46}$ mediante un gráfico de calibración, las diferencias absolutas entre la proporción de eventos esperados y observados fueron menores del $2 \%$ para los puntajes UDT-65 de $1 \mathrm{a}$ 4. El puntaje 0 del índice UDT-65 tuvo un comportamiento diferente al resto de los grupos, debido a que hubo una diferencia absoluta entre las proporciones, tanto esperada como observada, del $4.1 \%$, lo que podría explicarse por la baja frecuencia de enfermedad coronaria en población colombiana para ese grupo de pacientes y a la sobreestimación que hace el puntaje 0 del índice UDT-65 en nuestra población. Aun con estos resultados, que no superan el $5 \%$ en la diferencia absoluta, se decidió clínicamente que para el puntaje 0 el modelo está bien calibrado en nuestra población para este subgrupo, debido a que se espera que los pacientes sin factores de riesgo cardiovascular convencionales no presenten enfermedad coronaria. De igual forma, una prueba de ji al cuadrado con $p>0.05$ mostró que las proporciones obtenidas no fueron diferentes de aquellas esperadas para la enfermedad coronaria.

Como la capacidad de discriminación del índice UDT-65 para clasificar la enfermedad coronaria en población española obtuvo un AUC de 0.87 , se determinó desde el diseño de la investigación que en la población de estudio el índice UDT-65 tendría una adecuada discriminación si presentara un AUC de 0.80 o más. Es así como en la población colombiana se encontró una capacidad de discriminación adecuada con un AUC ROC de 0.8667 (IC95\%: 0.847410.88543 ), que se acerca al valor obtenido en la población española en la que fue desarrollado el índice. En ambas poblaciones, estos resultados son muy superiores a los encontrados en el estudio realizado por Sprockel, et al. ${ }^{47}$ en población colombiana, el cual midió la capacidad de discriminación de las escalas de riesgo HEART, TIMI, GRACE, CARdiac, Florencia y Sanchis, y las escalas HEART (AUC de 0.75; IC95\%: 0.69-0.81) y TIMI (AUC 0,71; IC95\%: 0.65-0.77) fueron las que presentaron el mejor rendimiento diagnósti$\mathrm{co}^{47-49}$. Estos resultados se explican, posiblemente, por el tipo de instituciones cardiovasculares especializadas donde se desarrolló y validó el índice UDT-65, lo que explicaría la mayor prevalencia de enfermedad coronaria y supone que la población evaluada puede influir en los resultados del mayor desempeño diagnóstico del índice en mención, en contraste con el menor desempeño presentado por las escalas evaluadas en población colombiana por Sprockel, et al. ${ }^{47}$ que, aunque provienen de instituciones de tercer nivel de complejidad, no son especializadas en el área cardiovascular.

\section{Conclusiones}

Teniendo en cuenta que la mitad de los pacientes que acuden a urgencias con dolor torácico tienen un riesgo bajo, los hallazgos de la validación del índice UDT-65 en la población de estudio sugieren que este 
modelo de predicción tipo diagnóstico es una buena opción para efectuar una aplicación válida en aquellos pacientes con dolor torácico no traumático de posible origen coronario que consulten al servicio de urgencias de una institución cardiovascular de referencia nacional, como la clínica en mención, debido a que mostró un buen rendimiento diagnóstico. Además, se determinó que el índice UDT-65 es un instrumento de fácil aplicación en el servicio de urgencias y no requiere mucho tiempo en su diligenciamiento.

\section{Limitaciones}

Pese a lo anterior, en población colombiana, diferente de la población de estudio, podría tener un menor rendimiento diagnóstico, quizá más cercano al mostrado por la escala HEART en población colombiana. Por ser un centro de referencia cardiovascular, la clínica Shaio tiene una gran población mayor de 65 años y con enfermedad coronaria previa, por lo que algunos resultados podrían no ser aplicables a otro tipo de población. Se requieren más estudios y la socialización de este índice en otras instituciones.

Es posible que algunos pacientes incluidos en el grupo de enfermedad coronaria no la tengan, 0 al contrario, debido a que no se dispone de arteriografía coronaria en todos los pacientes. La mayor limitación del estudio, que también se presentó en el estudio español, viene dada por la baja cantidad de pacientes con un puntaje de 4 en el índice UDT-65.

\section{Financiamiento}

Los autores declaran que no recibieron apoyo financiero para la publicación de este artículo.

\section{Conflicto de intereses}

Los autores declaran no tener conflicto de intereses.

\section{Responsabilidades éticas}

Protección de personas y animales. Los autores declaran que para esta investigación no se han realizado experimentos en seres humanos ni en animales.

Confidencialidad de los datos. Los autores declaran que han seguido los protocolos de su centro de trabajo sobre la publicación de datos de pacientes.
Derecho a la privacidad y consentimiento informado. Los autores declaran que en este artículo no aparecen datos de pacientes.

\section{Bibliografía}

1. Simoons ML. Cardiovascular disease in Europe: challenges for the medical profession: opening address of the 2002 Congress European Society of Cardiology. Eur Heart J. 2003;24:8-12.

2. Bayón J, Alegría E, Bosch X, Cabadés A, Iglesias I, Jiménez J, et al. Unidades de dolor torácico. Organización y protocolo para el diagnóstico de los síndromes coronarios agudos. Rev Esp Cardiol. 2002;55:143-54.

3. Alegría E, Bayón J. Chest pain units: a cardiologists' plead for its urgent implementation. Rev Esp Cardiol. 2002;55:1013-4.

4. Mendoza F. Guías colombianas de cardiología: síndrome coronario agudo con elevación del ST. Rev Colom Cardiol. 2010;17:123-65.

5. Mendoza F. Guías colombianas de cardiología: síndrome coronario agudo sin elevación del ST. Rev Colom Cardiol. 2008;15:145-62.

6. Ministerio de Salud y Protección Social. Situación de salud en Colombia: indicadores básicos 2016. Disponible en: www.minsalud.gov.co.

7. Sanz G. Estratificación del riesgo coronario en la unidad de dolor torácico: un problema no resuelto. Rev Esp Cardiol. 2005;58:772-4.

8. Steyerberg E. Clinical prediction models: a practical approach to development, validation, and updating. New York: Springer; 2009.

9. Altman DG, Royston $P$. What do we mean by validating a prognostic model? Statist Med. 2000;19:453-73.

10. Vergouwe Y, Steyerberg EW, Eijkemans MJ, Habbema JD. Substantial effective sample sizes were required for external validation studies of predictive logistic regression models. J Clin Epidemiol. 2005;58:475-83.

11. Martínez-Sellés M, Bueno H, Sacristán A, Estévez A, Ortiz J, Gallego L, et al. Dolor torácico en urgencias: frecuencia, perfil clínico y estratificación del riesgo. Rev Esp Cardiol. 2008;61:953-9.

12. Martínez-Sellés M, Ortiz J, Estévez A, Andueza J, de Miguel J, Bueno H. Un nuevo índice de riesgo para pacientes con ECG normal o no diagnóstico ingresados en la unidad de dolor torácico. Rev Esp Cardiol. 2005;58:782-8.

13. Geleijnse ML, Elhendy A, Kasparzak JD, Rambaldi R, Van Domburg RT, Cornel JH, et al. Safety and prognosis value of early dobutamine-atropine stress echocardiography in patients with spontaneous chest pain and a non-diagnostic electrocardiogram. Eur Heart J. 2000;21:397-406.

14. Moons KG, Royston P, Vergouwe Y, Grobbee DE, Altman DG. Prognosis and prognostic research: what, why, and how? BMJ. 2009;338:b375.

15. Steyerberg E. Clinical prediction models: a practical approach to development, validation, and updating. New York: Springer; 2009. p. 335-60.

16. Bachmann LM, Puhan MA, Ter Riet G, Bossuyt PM. Sample sizes of studies on diagnostic accuracy: literature survey. BMJ. 2006;332:1127-9.

17. Weber $\mathrm{C}$, Noels $\mathrm{H}$. Atherosclerosis: current pathogenesis and therapeutic options. Nat Med. 2011;17:1410-22.

18. Thygesen K, Alpert JS, Jaffe AS, Chaitman BR, Bax JJ, Morrow DA, et al. The Executive Group on behalf of the Joint European Society of Cardiology (ESC)/American College of Cardiology (ACC)/American Heart Association (AHA)/World Heart Federation (WHF) Task Force for the Universal Definition of Myocardial Infarction. Fourth universal definition of myocardial infarction (2018). Circulation. 2018;138:e618-51.

19. Lanas F, Avezum A, Bautista LE, Díaz R, Luna M, Islam S, et al. Risk factors for acute myocardial infarction in Latin America: the INTERHEART Latin American study. Circulation. 2007;115:1067-74.

20. Schargrodsky $H$, Hernández-Hernández $R$, Champagne BM, Silva $H$ Vinueza R, Silva Ayçaguer LC, et al. CARMELA: assessment of cardiovascular risk in seven Latin American cities. Am J Med. 2008;121:58-65.

21. Janssen KJ, Vergouwe $Y$, Donders AR, Harrell FE Jr, Chen $Q$, Grobbee DE, et al. Dealing with missing predictor values when applying clinical prediction models. Clin Chem. 2009;55:994-1001.

22. Steyerberg EW, Vickers AJ, Cook NR, Gerds T, Gonen M, Obuchowski N, et al. Assessing the performance of prediction models: a framework for traditional and novel measures. Epidemiology. 2010;21:128-38.

23. Muñoz OM, Rodríguez NI, Ruiz A, Rondón M. Validación de los modelos de predicción de Framingham y PROCAM como estimadores del riesgo cardiovascular en una población colombiana. Rev Colom Cardiol. 2014;21:202-12.

24. Moons KG, Kengne AP, Grobbee DE, Royston $P$, Vergouwe $Y$, Altman DG, et al. Risk prediction models: II. External validation, model updating, and impact assessment. Heart. 2012;98:691-98.

25. Hosmer DW, Taber S, Lemeshow S. The importance of assessing the fit of logistic regression models: a case study. Am J Public Health. 1991;81:1630-5

26. Jiang $X$, Osl M, Kim J, Ohno-Machado L. Calibrating predictive model estimates to support personalized medicine. J Am Med Inform Assoc. 2012;19:263-74. 
Rev Colomb Cardiol. 2021;28(5)

27. Cook NR. Statistical evaluation of prognostic versus diagnostic models: beyond the ROC curve. Clin Chem. 2008;54:17-23.

28. Cook NR. Use and misuse of the receiver operating characteristic curve in risk prediction. Circulation. 2007:115:928-35.

29. Declaración de Helsinki de la Asociación Médica Mundial sobre principios éticos para las investigaciones médicas en seres humanos. Traducción castellana. Universidad de Navarra: Centro de Documentación de Bioética: 2008.

30. Resolución 8430 de 1993: Normas científicas, técnicas y administrativas para la investigación en salud. Ministerio de Salud de Colombia; 1993.

31. Panamerican Health Organization. Health in the Americas; 2017 Edition. Summary: Regional Outlook and Country Profiles. Washington, D.C. PAHO; 2017.

32. Than M, Cullen L, Reid C, Lim SH, Aldous S, Ardagh MW, et al. A 2-h diagnostic protocol to assess patients with chest pain symptoms in the Asia-Pacific region (ASPECT): a prospective observational validation study. Lancet. 2011;377:1077-84.

33. Bedoya-Ríos C, Mendoza-Lozano J, Nieto O. Prevalencia de infarto de miocardio en un programa de riesgo cardiovascular de una institución prestadora de salud en Armenia-Quindío. Rev Colom Cardiol. 2016; 23:561-7.

34. National Cholesterol Education Program Adult Treatment Panel III: Third Report of the National Cholesterol Education Program (NCEP) Exper Panel on Detection, Evaluation, and Treatment of High Blood Cholesterol in Adults (Adult Treatment Panel III) final report. Circulation. 2002;106:3143-21.

35. American Diabetes Association. Diagnosis and classification of diabetes mellitus. Diabetes Care. 2011;34 (Suppl 1):S62-9.

36. World Conference on Tobacco or Health. Tobacco control country profiles. American Cancer Society, World Health Organization, and International Union Against Cancer; 2003.

37. Chobanian AV, Bakris GL, Black HR, Chobanian AV, Bakris GL, Black HR, et al. The Seventh Report of the Joint National Committee on Prevention, Detection, Evaluation, and Treatment of High Blood Pressure: the JNC 7 report. JAMA. 2003;289:2560-72
38. Streiner DL. A checklist for evaluating the userfulness of raiting scales. Can J Psychiatry. 1993;38:140-8.

39. Roger VL. Epidemiology of myocardial infarction. Med Clin North Am. 2007:91:537-52.

40. Singh M. Risk stratification following acute myocardial infarction. Med Clin North Am. 2007;91:603-16.

41. Morrow DA, Antman EM, Giugliano RP, Cairns R, Charlesworth A, Murphy SA, et al. A simple risk index for rapid initial triage of patients with ST-elevation myocardial infarction: an InTIME II substudy. Lancet. 2001;358:1571-5.

42. Antman EM, Cohen M, Bernink PJ, McCabe $\mathrm{CH}$, Horacek $\mathrm{T}$, Papuchis G, et al. The TIMI risk score for unstable angina/non-ST elevation Ml: a method for prognostication and therapeutic decision making. JAMA. 2000;284:835-42.

43. Eagle KA, Lim MJ, Dabbous OH, Pieper KS, Goldberg RJ, Van de Werf $F$, et al. A validated prediction model for all forms of acute coronary syndrome: estimating the risk of 6-month postdischarge death in an international registry. JAMA. 2004;291:2727-33.

44. Damen JAAG, Hooft L, Schuit E, Debray TPA, Collins GS, Tzoulaki I, et al. Prediction models for cardiovascular disease risk in the general population: systematic review. BMJ. 2016;353:i2416.

45. Altman DG, Vergouwe $Y$, Royston $P$, Moons KG. Prognosis and prognostic research: validating a prognostic model. BMJ. 2009;338:b605

46. Vergouwe Y, Royston P, Moons KG, Altman DG. Development and validation of a prediction model with missing predictor data: a practical approach. J Clin Epidemiol. 2010;63:205-14.

47. Sprockel JJ, Mantilla H, Cruz L, Barón R, Diaztagle J. Aplicación de las escalas de estratificación del riesgo en el diagnóstico de los síndromes coronarios agudos. Rev Colom Cardiol. 2017;24:480-7.

48. Ruiz A. Uso de pruebas diagnósticas en medicina clínica. En: Ruiz A, Morillo L, editores. Epidemiología clínica: investigación clínica aplicada. Bogotá: Médica Panamericana; 2009. p. 111-28.

49. Beck JR, Shultz EK. The use of relative operating characteristic (ROC) curves in test performance evaluation. Arch Pathol Lab Med. 1986; 110:13-20. 